\title{
Right atrium tumor - pseudoaneurysm of right coronary artery. A rare complication after percutaneous coronary intervention
}

\author{
Andrzej Łoś ${ }^{1}$, Radosław Nowak ${ }^{1}$, Maksymilian Mielczarek ${ }^{2}$, Radosław Kozaryn ${ }^{1}$, Dariusz Jagielak ${ }^{1}$, \\ Piotr Siondalski ${ }^{1}$ \\ ${ }^{1}$ Cardiac Surgery Department, Medical University of Gdansk, Gdansk, Poland \\ ${ }^{2}$ First Cardiology Department, Medical University of Gdansk, Gdansk, Poland
}

Adv Interv Cardiol 2017; 13, 4 (50): 341-342

DOI: https://doi.org/10.5114/aic.2017.71619

Cardiac masses include tumors, thrombi, vegetations, calcific lesions and other rare conditions [1]. An increasing number of reports describe pseudoaneurysm formation as a complication of percutaneous coronary interventions $(\mathrm{PCl})$. Such a pseudoaneurysm may potentially create a bulge that mimics a cardiac mass. Therefore, it is necessary to include pseudoaneurysm in the differential diagnosis of cardiac masses.

We present a case of a 56-year old man, who was referred for a right atrial mass that might have corresponded to a myxoma on the basis of its location and characteristics of a cyst. The patient presented symptoms of stable coronary artery disease (CAD) in class III of the CCS scale. Several years previously the patient underwent two PCls within the right coronary artery (RCA). Initially a bare-metal stent (BMS) was implanted, and a couple of months later a drug-eluting stent (DES) was deployed within the BMS due to diffuse restenosis. Both procedures in the RCA were uncomplicated with no extravasation of contrast dye. Apart from CAD, the medical record included thrombocytopenia.

Prior to surgery, transthoracic echocardiography (TTE), cardiac magnetic resonance (CMR) and coronary angiography (CA) were conducted to determine the diagnosis for the round mass. The TTE revealed a spherical, calcified mass in the right atrium (RA) of $3 \times 3 \times 2 \mathrm{~cm}$ in dimensions (Figure $1 \mathrm{~A})$. Coronary angiography revealed significant stenosis in the RCA (in-stent restenosis) (Figure $1 \mathrm{~B}$ ) as well as in the left anterior descending (LAD). Cardiac magnetic resonance confirmed the presence of a tumor in the RA in direct communication with the RCA the suspicion of pseudoaneurysm was made (Figure $1 \mathrm{C}$ ). The differential diagnosis included myxoma, thrombus and echinococcal infection. The patient was referred to the operating theater.

During the surgery the patient underwent resection of the pseudoaneurysm with the use of cardiopulmonary bypass and bicaval cannulation. A $2 \times 3 \times 2 \mathrm{~cm}$ oval tumor turned out to be an old round hematoma with signs of fibrosis and calcifications surrounding the RCA with the stent inside (Figure 1 D). Concomitant myocardial revascularization of the RCA was performed using a saphenous vein graft and the LAD was grafted with the left internal mammary artery.

The postoperative period was uneventful. The patient continues to do well with subsequent follow-ups. Histological evaluation revealed a thrombus with advanced fibrosis.

Atherosclerosis or drug-eluting stents are probable causes of coronary aneurysm. The drug-eluting stent contains immunosuppressants, such as sirolimus, or chemotherapeutic agents like paclitaxel, which is an anti-inflammatory agent and inhibits cell proliferation [2].

Coronary aneurysm formation is well recognized after stent implantation, and is observed much more frequently after DES implantation than after BMS implantation [3].

The proposed pathogenesis of PCI-related pseudoaneurysm might be multifactorial [4]. It may be hypothesized that long-lasting friction motion of the stiff double-metal layer against the arterial wall at the proximal edge of the stented segment resulted in artery tear, subsequent blood extravasation and pseudoaneurysm formation, which finally healed spontaneously. In this case, friction motion might have been exacerbated by a bend of the artery at the proximal edge of the stented segment. This is supported by the presence of severe edge restenosis at the site, which reflects intense local inflammation.

\section{Corresponding author:}

Andrzej Łoś MD, Cardiac Surgery Department, Medical University of Gdansk, 7 Dębinki St, 80-211 Gdansk, Poland, phone: +48 583417669 , e-mail: alos@gumed.edu.pl

Received: 25.09.2017, accepted: 30.10.2017. 

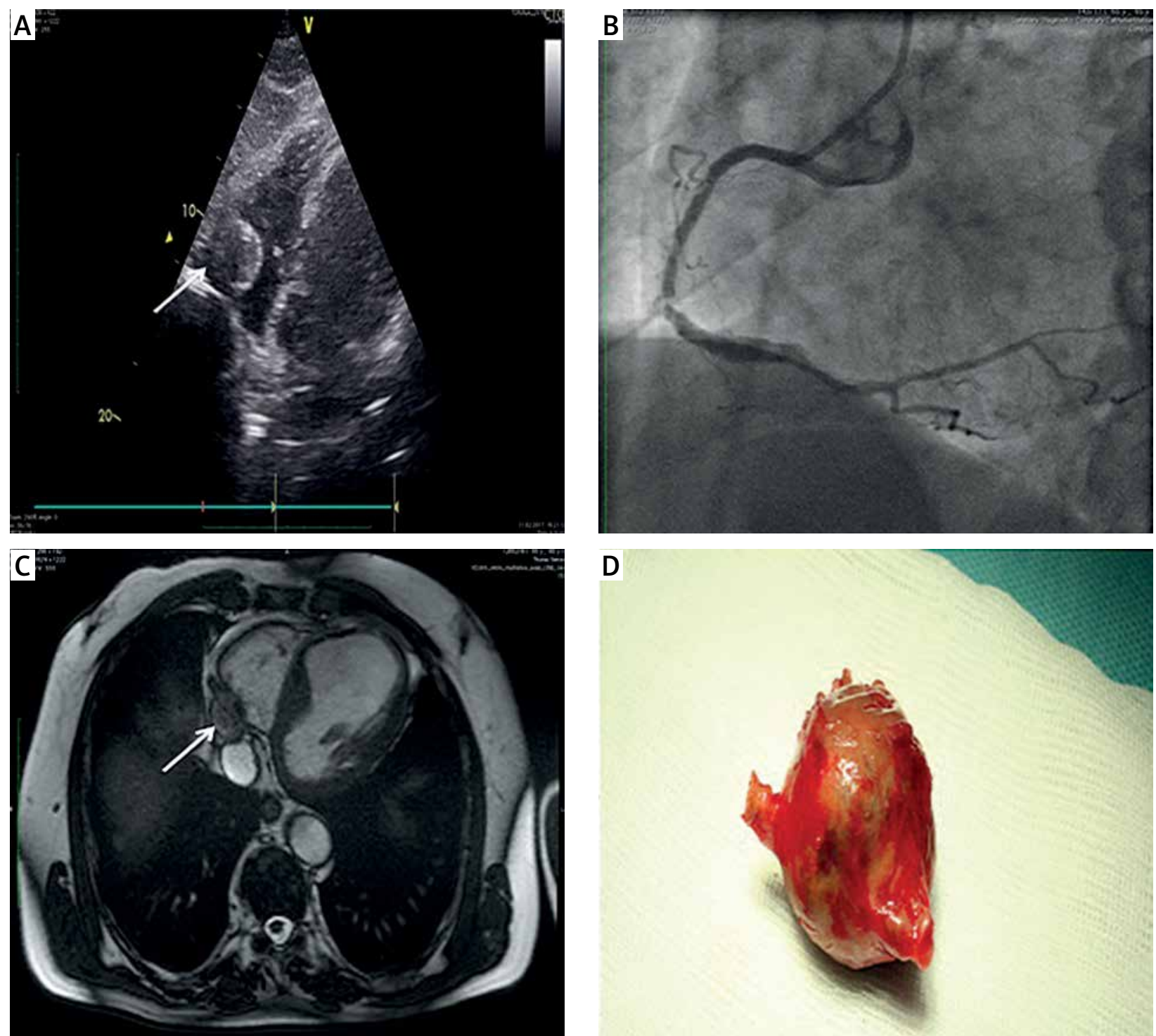

Figure 1. A - Transthoracic echocardiography in 4-chamber subcostal projection showing an oval tumor in the

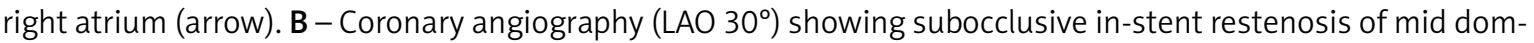
inant right coronary artery. C - Cardiac MRI showing abnormal mass in right atrium connected with coronary artery (arrow). D - Postoperative photography of pseudoaneurysm of the right coronary artery

In this patient a low platelet count might also be responsible for pseudoaneurysm formation.

In general, procedural complications in the form of local dissection or an extraluminal dye deposit can be considered as a point of origin for a pseudoaneurysm.

Finally, we suggest implanting stents along a bend of an artery rather than on its verge, as this may reduce the risk of arterial injury by stiff struts, particularly when a second stent needs to be deployed to address in-stent restenosis. We believe that this attitude may help to prevent edge restenosis and pseudoaneurysm formation.

\section{Conflict of interest}

The authors declare no conflict of interest.

\section{References}

1. Basso C, Rizzo S, Valente M, et al. Cardiac masses and tumours. Heart 2016; 102: 1230-45.

2. Vassilev D, Karamfiloff K, Gil R. Aneurysm formation after paclitaxel-eluting balloon angioplasty used to treat coronary restenosis after plain old balloon angoplasty - case report and review of the literature. Postep Kardiol Interw 2015; 11: 250-1.

3. Tirani HD, Aghajanzadeh M, Pourbahador R, et al. Giant right coronary aneurysm mimicking a mediastinal cyst with compression effect: a case report. Res Cardiovasc Med 2016; 5: 32086.

4. Kawai Y, Kitayama M, Akao H, et al. A case of coronary rupture and pseudoaneurysm formation after fracture of implanted paclitaxel-eluting stents. Cardiovasc Interv Ther 2016; 31: 231-7. 